\title{
AN ANALYTIC SET-VALUED SELECTION AND ITS APPLICATIONS TO THE CORONA THEOREM, TO POLYNOMIAL HULLS AND JOINT SPECTRA
}

\author{
BY
}

ZBIGNIEW SLODKOWSKI

\begin{abstract}
It is shown that for every annulus $P=\left\{z \in \mathbf{C}^{n}: \delta<|z|<r\right\}$, $\delta>0$, there exists a set-valued correspondence $z \rightarrow K(z): P \rightarrow 2 \mathbf{C}^{n}$, whose graph is a bounded relatively closed subset of the manifold $\{(z, w) \in P \times$ $\left.\mathbf{C}^{n}: z_{1} w_{1}+\cdots+z_{n} w_{n}=1\right\}$ which can be covered by $n$-dimensional analytic manifolds.

The analytic set-valued selection $K$ obtained thereby is then applied to several problems in complex analysis and spectral theory which involve solving the equation $a_{1} x_{1}+\cdots+a_{n} x_{n}=y$. For example, an elementary proof is given of the following special case of a theorem due to Oka: every bounded pseudoconvex domain in $\mathbf{C}^{2}$ is a domain of holomorphy.
\end{abstract}

0. Introduction. Consider the family of complex hyperplanes

$$
L_{z}=\left\{w \in \mathbf{C}^{n}: z_{1} w_{1}+\cdots+z_{n} w_{n}=1\right\}, \quad z \in \mathbf{C}^{n} \backslash\{0\} .
$$

By Hartogs's theorem there does not exist a single-valued analytic selection from $\left\{L_{z}\right\}$ over the annulus

$$
P_{\delta, r}=\left\{z \in \mathbf{C}^{n}: \delta<|z|<r\right\} .
$$

Yet a need of such a selection or a good substitute is apparent in connection with various questions of spectral theory, since solving the equation $a_{1} x_{1}+\cdots+a_{n} x_{n}=e$ in a suitable Banach algebra or module is all that spectral theory is about.

In $[11, \S 4]$ the author has constructed a set-valued selection from $\left\{L_{z}\right\}_{z}$ over $P$, analytic in the sense explained below, and obtained some interesting applications of it $[\mathbf{1 1}, \S \S 5-6]$.

The purpose of this paper is to extend the construction of this selection to arbitrary dimension and to apply the obtained multifunction to several topics in complex analysis and spectral theory. for.

The following theorem states the basic properties of the selection we are looking

THEOREM 0.1. Let $0<\delta<r$ and $n$ be an integer. Then there exists a setvalued function $z \rightarrow K(z): P_{\delta, r} \rightarrow 2^{\mathrm{C}^{n}}$, such that $K(z) \subset L_{z}$ for every $z \in P_{\delta, r}$, which is analytic in the sense of the next definition.

DEFINITION $0.2[\mathbf{1 2}, \S 2]$. Let $G \subset \mathbf{C}^{k}$ be open. An upper semicontinuous setvalued function (shortly: multifunction) $z \rightarrow K(z): G \rightarrow 2^{\mathrm{C}^{n}}$ is called analytic

Received by the editors May 15, 1985 . 47A10.

1980 Mathematics Subject Classification. Primary 32A30, 32E20, 46J15; Secondary 32D15, 
if for every $(n+1)$-dimensional complex plane $L \subset \mathbf{C}^{k+n}$, the intersection $Y=$ $L \cap\{(z, w): z \in G, w \in K(z)\}$ has local maximum property in the following sense: there does not exist a function $f(z, w)$ analytic in a neighbourhood of a point $\left(z^{*}, w^{*}\right)$ such that $|f| \mid Y$ has strict local maximum at $\left(z^{*}, w^{*}\right)$.

The reader is referred for background and further information on analytic multifunctions to $[3-4,8-10$ and 11-15].

1. An analytic set-valued selection. Similarly as in $[11, \S 4]$ we are looking for a set-valued selection of the form

$$
K(z)=\left\{x \in \mathbf{C}^{n}:\langle z, w\rangle=1,\left.|w-\bar{z}| z\right|^{-2} \mid \leq e^{\rho(\log |z|)},\right.
$$

where $z \in P_{\delta, r}$ and $\rho:(\log \delta, \log r) \rightarrow R$ is a smooth function. $(\langle z, w\rangle$ denotes the bilinear form $z_{1} w_{1}+\cdots+z_{n} w_{n}$.) The motivation is that both the manifold $\langle z, w\rangle=1$ and the graph of $K$,

$$
Y=\left\{(z, w) \in P_{\delta, r} \times \mathbf{C}^{n}: w \in K(z)\right\},
$$

are preserved by the group $G$ consisting of biholomorphic maps

$$
(z, w) \rightarrow(A z, \bar{A} w): \mathbf{C}^{2 n} \rightarrow \mathbf{C}^{2 n},
$$

where $A$ is any unitary $n \times n$ matrix.

We will prove now the lemma which gives a sufficient condition for the multifunction (1.1) to be analytic.

LEMMA 1.1. Let $0<\delta<r$ and let $\rho:(\log \delta, \log r) \rightarrow R$ be a convex, $C^{(2)}$ smooth function satisfying the inequality

$$
\rho^{\prime \prime}(x)\left(e^{2 \rho(x)+2 x}\left(\rho^{\prime}(x)-1\right)-2\right)>2\left(\rho^{\prime}(x)+1\right)^{2} .
$$

Then the graph $Y$ of the multifunction $K: P_{\delta, r} \rightarrow 2^{\mathbf{C}^{n}}$, defined by (1.1) can be covered by $n$-dimensional analytic manifolds.

PROOF. The case $n=2$ of this lemma was proved in [11, Theorem 4.3 and Lemma 4.4]. (The reader will note a misprint in equation (4.7) in [11].) We have shown that the set $\left\{(z, w) \in \mathbf{C}^{4}: \delta<|z|<r, w \notin K(z)\right\}$ is strictly pseudoconvex in $\{(z, w): \delta<|z|<r\}$ and so, as is well known, its complement can be covered by (open pieces of) two-dimensional analytic manifolds.

Decompose $\mathbf{C}^{2 n}=H \oplus V$, where $V=\left\{z_{1}=z_{2}=w_{1}=w_{2}=0\right\}$ and $H$ is its orthogonal complement. Since $Y \cap H$ is the graph of the multifunction (1.1) for $n=2$, for every $p \in H \cap Y$ there is a two-dimensional complex manifold $M$ such that $p \in M \subset H \cap Y$ and $M$ is relatively compact in $Y$.

ASSERTION. For fixed $M$ there is $\varepsilon>0$ such that $M \times B_{n-2}(\varepsilon) \subset Y$ where $B_{n-2}(\varepsilon)=\left\{u \in \mathbf{C}^{n-2}:|u|<\varepsilon\right\}$.

To prove this let

$$
F(z, w)=R\left(|z|^{2}\right)-|w|^{2}+|z|^{-2}
$$

where $R(t)=\exp \left(2 \rho\left(\frac{1}{2} \log t\right)\right)$. Then the set $Y$ is defined by the relations

$$
F(z, w) \geq 0, \quad\langle z, w\rangle=1, \quad \delta<|z|<r .
$$

Let us represent points of $M$ as $(a, 0, b, 0) \in \mathbf{C}^{2 n}$; we have to show that $F(a, u, b, 0)$ $\geq 0$ for $u \in \mathbf{C}^{n-2}$, with $|u|$ small enough. Set

$$
f_{a, b}(u)=F(a, u, b, 0)=R\left(|a|^{2}+|u|^{2}\right)-|b|^{2}+\left(|a|^{2}+|u|^{2}\right)^{-1} .
$$


Since $R(t)=R\left(|a|^{2}\right)+R^{\prime}\left(|a|^{2}\right)\left(t-|a|^{2}\right)+o\left(t-|a|^{2}\right)$,

$$
f_{a, b}(u)=\left(R\left(|a|^{2}\right)-|b|^{2}-|a|^{-2}\right)+\left(R^{\prime}\left(|a|^{2}\right)-|a|^{-4}\right)|u|^{2}+o\left(|u|^{2}\right) .
$$

The scalar $R^{\prime}\left(|a|^{2}\right)-|a|^{-4}$ is positive. (Indeed,

$$
\begin{aligned}
R^{\prime}\left(|a|^{2}\right)-|a|^{-4} & =|a|^{-2}\left(e^{2 \rho(\log |a|)} \rho^{\prime}(\log |a|)-|a|^{-2}\right) \\
& =e^{-2 x}\left(e^{-2 x} D+e^{2 \rho(x)}+e^{-2 x}\right),
\end{aligned}
$$

for $x=\log |a|$, and $D=\left(\rho^{\prime}(x)-1\right) \exp (2 \rho(x)+2 x)-2$ is positive by assumption (1.4).)

Thus the function $f_{a, b}(u)$ is convex in some ball $B_{n-2}(\varepsilon)$, and by smoothness of $F$ and relative compactness of $M$ in $Y, \varepsilon>0$ can be chosen independently on $(a, b) \in M$. Furthermore by $(1.7) \operatorname{grad} f_{a, b}(0)=0$. Therefore

$$
f_{a, b}(u) \geq f_{a, b}(0)=F(a, 0, b, 0) \geq 0,
$$

for $(a, b) \in M,|u|<\varepsilon$, and so $(a, u, b, 0) \in Y$, which settles the assertion.

To complete the proof of the lemma observe that every point $q \in Y$ is the image by a biholomorphic map $g_{A} \in G$ (cf. (1.3)) of some point $p \in H \cap Y$ and so $q \in g_{A}\left(M \times B_{n-2}(\varepsilon)\right) \subset Y$. Q.E.D.

REMARK. The lemma can also be checked directly by parametrizing the manifold $\langle z, w\rangle=1$ (for $z_{1} \neq 0$ ) by $z_{1}, \ldots, z_{n}, w_{2}, \ldots, w_{n}$ and substituting $w_{1}=z_{1}^{-1}-$ $z_{1}^{-1}\left(z_{2} w_{2}+\cdots+z_{n} w_{n}\right)$ into (1.5). Direct computation of the Hessian of the resulting function at points with $z_{2}=\cdots=z_{n}=w_{3}=\cdots=w_{n}=0$ yields fairly quickly condition (1.4).

REMARK 1.2. We have to give an example of the function $\rho(\cdot)$. By substituting $\rho(x)=\frac{1}{2} f(4 x)-x$ we reduce inequality (1.4) to the simple form

$$
f^{\prime \prime}\left(e^{f}\left(f^{\prime}-1\right)-1\right)>\frac{1}{2}\left(f^{\prime}\right)^{2} .
$$

The function $f(x)=\frac{1}{2}(x-a)^{2}+(x-a)+2$ satisfies this inequality in $(a, \infty)$.

The relation of Lemma 1.1 to Definition 0.1 and to another notion of analyticity of set-valued functions is discussed in $\S 3$.

2. A proof of the corona theorem for $n$ generators. The following theorem was proven independently and in different ways by Alexander and Wermer [2] and by the author [15].

THEOREM 2.1. Let $X$ be a compact subset of $\partial D \times \mathbf{C}^{n}(D$ denotes the unit disc). Assume that for every $z \in \partial D$ the section $X_{z}=\left\{w \in \mathbf{C}^{n}:(z, w) \in X\right\}$ is convex. Then for every point $\left(z^{*}, w^{*}\right)$ in the polynomially convex hull $h(X)$ of $X$ there exists a bounded analytic function $g: D \rightarrow \mathbf{C}^{n}$ such that $g\left(z^{*}\right)=w^{*}$ and $(z, g(z)) \in h(X)$ for all $z \in D$.

This theorem and the next proposition (cf. [11, Theorem 3.2] for $n=1$ and [13, Proposition 2.3] for arbitrary $n$ ) are the basis of the recent approach to the corona theorem due to Berndtsson and Ransford [6].

Proposition 2.2. Let $L: \bar{G} \rightarrow 2^{\mathrm{C}^{n}}$, where $G$ is a bounded planar domain, be an upper semicontinuous multifunction whose restriction to $G$ is analytic. Then the graph of $L$ is contained in the polynomial hull of the set $X=\{(z, w): z \in \partial G, w \in$ $L(z)\}$.

(The proof is sketched at the end of this section.) 
COROllaRY $2.3[6]$. Let $L: \bar{D} \rightarrow 2^{\mathrm{C}^{n}}$ be an upper semicontinuous multifunction whose restriction to $D$ is analyticc. Then there exists a bounded analytic function $g: D \rightarrow \mathbf{C}^{n}$ whose nontangential limit $\tilde{g}\left(e^{i \theta}\right)$ belongs to $L\left(e^{i \theta}\right)$ for almost all $\theta$.

Berndtsson and Ransford [6] made an important observation that this fact, which is a direct consequence of Theorem 2.1 and Proposition 2.2, is all that is needed to get a proof of the corona theorem, provided a suitable multifunction $L$ is constructed. In this way they obtained a new proof of Wolff's $\bar{\partial}$-theorem (and thereby a new proof of the corona theorem). After being informed about this proof, the author has pointed out that Corollary 2.3 and [11, Theorem 4.3] yield a direct proof of the corona theorem without the $\bar{\partial}$-equation (in case of two generators). Subsequently Berndtsson and Ransford obtained another direct proof (in the same case) $[6, \S 2]$, and the author applied his argument to an arbitrary number of generators, as presented here. (See also final remarks in Berndtsson and Ransford [6, §3].)

Let $f_{1}, \ldots, f_{n} \in H^{\infty}(D)$ satisfy the inequality

$$
\delta^{2}<\left|f_{1}(z)\right|^{2}+\cdots+\left|f_{n}(z)\right|^{2}<1, \quad z \in D .
$$

We are looking for $g_{1}, \ldots, g_{n} \in H^{\infty}(D)$ satisfying

$$
g_{1}(z) f_{1}(z)+\cdots+g_{n}(z) f_{n}(z)=1, \quad z \in D
$$

and a uniform estimate

$$
\sup _{|z|<1}\left(\left|g_{1}(z)\right|^{2}+\cdots+\left|g_{n}(z)\right|^{2}\right)<C_{\delta}^{2}
$$

where $C_{\delta}$ depends only on $\delta$. We can assume without loss of generality that $f_{1}, \ldots, f_{n}$ are continuous on $\bar{D}$. Set $f(z)=\left(f_{1}(z), \ldots, f_{n}(z)\right)$. Then $f(D) \subset$ $P_{\delta}=\left\{z \in \mathbf{C}^{n}: \delta<|z|<1\right\}$.

LEMMA 2.4. Let $K: P_{\delta} \rightarrow 2^{\mathrm{C}^{n}}$ be any multifunction satisfying the conditions of Lemma 1.1. Then the multifunction $L(z)=K(f(z)), z \in D$, is analytic in $D$.

ProOF. Fix $z^{*} \in D$ and $w^{*} \in L(z)$. Then $\left(f\left(z^{*}\right), w^{*}\right) \in Y(=\operatorname{gr}(K))$. By Lemma 1.1 there exists an $n$-dimensional analytic manifold $M$ such that $\left(f\left(z^{*}\right), w^{*}\right)$ $\in M \subset Y, M$ is locally given by $n$ analytic equations $g_{i}(u, w)=0, i=1, \ldots, n$, $(u, z) \in \mathbf{C}^{2 n}$. Let

$$
N=\left\{(z, w) \in \mathbf{C} \times \mathbf{C}^{n}: g_{i}(f(z), w)=0,1 \leq i \leq n\right\} .
$$

Then $N$ is an analytic variety such that $\left(z^{*}, w^{*}\right) \in N \subset \operatorname{gr}(L)$ and has dimension $\geq 1$ at $\left(z^{*}, w^{*}\right)$. Observe then that for no analytic function $h(z, w)$ defined in a neighborhood of $\left(z^{*}, w^{*}\right)$ can $|h|$ restricted to $\operatorname{gr}(L)$ have strict maximum at $\left(z^{*}, w^{*}\right)$, for this would violate the open mapping theorem for the function $h \mid N$ (by [7, Proposition 10, p. 54]). Q.E.D.

(A more elementary but longer proof follows from [11, Proposition 5.1]; cf. also discussion in $\S 3$.)

PROOF OF THE CORONA THEOREM. We construct the multifunction $L$ as in Lemma 2.4, assuming in addition that the function $\rho$ is continuous in $[\log \delta, 0]$. Then $L$ satisfies the uniform bound $\sup \{|w|: z \in D, w \in L(z)\} \leq C_{\delta}$. Applying 
Corollary 2.3 to $L$, we obtain a bounded analytic function $g: D \rightarrow \mathbf{C}^{n}$ such that $\tilde{g}\left(e^{i \theta}\right) \in L\left(e^{i \theta}\right)$ for a.a. $\theta$. By construction of $K(\mathrm{cf} .(1.1))$ this means that

$$
\left\langle\tilde{f}\left(e^{i \theta}\right), \tilde{g}\left(e^{i \theta}\right)\right\rangle=1 \quad \text { a.a. } \theta
$$

which implies (2.2). Of course

$$
\sup _{z \in D}|g(z)| \leq \sup _{\theta}\left|\tilde{g}\left(e^{i \theta}\right)\right| \leq C_{\delta} . \quad \text { Q.E.D. }
$$

Proof OF Proposition 2.2 (SKETCH). If not, then there is a polynomial $p(z, w)$ and $\varepsilon>0$ such that $\max u|\operatorname{gr}(L)>\max u| X$, where $u(z, w)=|p(z, w)|^{2}+$ $\varepsilon\left(|z|^{2}+|w|^{2}\right)$. Let $\left(z^{*}, w^{*}\right)$ be any point at which $u \mid \operatorname{gr}(L)$ attains its maximum; $z^{*} \in G$. Using the complex Taylor formula at $\left(z^{*}, w^{*}\right)$ we can write

$$
u(z, w)=\operatorname{Re} p(z, w)+h(z, w)+r_{2}(z, w)
$$

where $p(z, w)$ is an analytic polynomial with $\operatorname{deg} p \leq 2, h$ is a strictly positive homogeneous function in $z-z^{*}, w-w^{*}$ of degree 2 (i.e., Hessian) and $r_{2}=$ $o\left(\left|z-z^{*}\right|^{2}+\left|w-w^{*}\right|^{2}\right)$. Therefore the restriction of $\operatorname{Re} p=u-h-r_{2}$ to $\operatorname{gr}(L)$ has strict local maximum at $\left(z^{*}, w^{*}\right)$. Thus Definition 0.2 fails for $\exp (p(z))$. Q.E.D.

3. Approximation of analytic multifunctions by projections of polynomial hulls. In this section we apply the set-valued selection of $\S 1$ to prove the following result.

THEOREM 3.1. Let $G$ be a bounded planar domain and $K: G \rightarrow 2^{\mathrm{C}^{n}}$ an analytic multifunction with bounded graph. Then there exists a sequence of upper semicontinuous multifunctions $K^{s}: \bar{G} \rightarrow 2^{\mathbf{C}^{n}}, s \geq 1$, analytic in $G$ and such that

$$
K^{s+1}(z) \subset K^{s}(z) ; \quad K(z)=\bigcap_{s \geq 1} K^{s}(z) ;
$$

furthermore, for every $s$ there is an integer $m=m(s)$ and a compact subset $X^{s}$ of $\partial G \times \mathbf{C}^{n+m}$ such that $\operatorname{gr}\left(K^{s}\right)=\pi\left(h\left(X^{s}\right)\right)$, where $\pi: \mathbf{C}^{n+m+1} \rightarrow \mathbf{C}^{n+1}$ is the standard projection.

For the purpose of further discussion we will compare first Definition 0.2 with a weaker notion.

Definition $3.2[\mathbf{1 1}$, Definition $4.1 ; 12, \S 2]$. Let $G$ be an open subset of $\mathbf{C}^{k}$. An upper semicontinuous function $K: G \rightarrow 2^{\mathbf{C}^{n}}$ is said to be weakly analytic if for every open subset $G^{\prime}$ of $G$ and for every plurisubharmonic function $u(z, w)$ defined in the neighbourhood of $\operatorname{gr}\left(K \mid G^{\prime}\right)$ the function $v(z)=\max \{u(z, w): w \in K(z)\}$ is plurisubharmonic in $G^{\prime}$.

It turns out that the two notions are equivalent in case $k=1$ but not in general.

EXAMPLE 3.3. Let $K(z)=\partial D$ for $z \neq 0, z \in \mathbf{C}^{2}$ and $K(0)=\bar{D}$. Then $K: \mathbf{C}^{2} \rightarrow 2^{\mathbf{C}}$ fulfills Definition 3.2. However, hyperplane $\{w=0\}$ intersects $\operatorname{gr}(K)$ at a singleton which does not have the local maximum property.

PROPOSITION 3.4. Let $G$ be a planar domain and $K: G \rightarrow 2^{\mathrm{C}^{n}}$ an upper semicontinuous multifunction. Then $K$ is analytic if and only if for every plurisubharmonic function $u(z, w)$ in a neighbourhood of $\operatorname{gr}(K)$ the function

$$
v(z)=\max \{u(z, w): w \in K(z)\}
$$

is subharmonic in $G$. 
Proof (Sketch). Necessity (cf. [11, Theorem 3.2 for $n=1]$ ). First take $u$ strictly plurisubharmonic in a neighbourhood of $\operatorname{gr}\left(K \mid G^{\prime}\right)$, where $G^{\prime} \subset G$, and let $\bar{D}$ be a closed disc in $G^{\prime}$. The same argument as in the proof of Proposition 2.2 shows that

$$
\max u|\operatorname{gr}(K \mid \bar{D}) \leq \max u| \operatorname{gr}(K \mid \partial D)),
$$

and so $\max v|\bar{D} \leq \max v| \partial D$. Replacing $u(z, w)$ by $u(z, w)+\operatorname{Re} p(z)$, where $p(z)$ is a complex polynomial, we get

$$
\max (v+\operatorname{Re} p)|\bar{D} \leq \max (v+\operatorname{Re} p)| \partial D,
$$

for every $p$ and $D$, which implies that $v$ is subharmonic by $[\mathbf{5}, \S 4]$.

Sufficiency. Suppose $f(z, w)$ is analytic in a neighbourhood of a closed ball $\bar{B}$ centered at $\left(z^{*}, w^{*}\right)$ and $|f| \mid \operatorname{gr}(K) \cap \bar{B}$ has strict maximum at $\left(z^{*}, w^{*}\right)$. In the same way as in $[13$, Proof of Theorem 5.1] we find an open set $U$ and a number $d<\left|f\left(z^{*}, w^{*}\right)\right|$ such that $\left(z^{*}, w^{*}\right) \notin \bar{U},|f| \mid \partial B<d, \operatorname{gr}(K) \subset U \cup B$, and the function

$$
u(z, w)= \begin{cases}\max (|f|, d), & (z, w) \in \bar{B}, \\ d, & (z, w) \in U \backslash \bar{B},\end{cases}
$$

is plurisubharmonic in $U \cup B$. Then the function defined by (3.2) has strict maximum at $z^{*}$ and is not subharmonic. Q.E.D.

REMARK 3.5. By the last result and [11, Proposition 4.2(iii)] it suffices to consider only $G^{\prime}=G$ in Definition 3.2.

Remark 3.6. By Proposition 3.4 and [11, Proposition 4.2(iii)] every analytic multifunction $K: G \rightarrow 2^{\mathrm{C}^{n}}, G \subset \mathbf{C}^{k}$, is weakly analytic. (Consider a complex line $L$ in $\mathbf{C}^{k}$ and note that $\operatorname{gr}(K \mid L)=\operatorname{gr}(K) \cap\left(L \times \mathbf{C}^{n}\right)$.)

REMARK 3.7. The selection of Lemma 1.1 is analytic and so weakly analytic. (Apply [7, Proposition 10, p. 54] as in the proof of Lemma 2.4 or [13, Proposition $3.5(\mathrm{~b})]$ and note that by $[\mathbf{1 3}$, Corollary 2.6$]$ the class of $k$-maximum sets is preserved by biholomorphic maps.)

In the proof of Theorem 3.1 we use the fact that composition of weakly analytic multifunctions is weakly analytic $[\mathbf{1 1}$, Proposition 5.1]. (Note that Lemma 2.4 was a special instance of this.) The corresponding result for analytic multifunctions, which is less elementary, will appear in a subsequent paper.

ProOF OF THEOREM 3.1. This proof is an extension of $[\mathbf{1 1}, \S 5$, Proof of Theorem III]. Consider an annulus

$$
P=\left\{(z, w) \in \mathbf{C}^{n+1}: \delta^{2}<\left|z-z^{*}\right|^{2}+\left|w-w^{*}\right|^{2}<r^{2}\right\}
$$

containing $\operatorname{gr}(K)$. Let $L: P \rightarrow 2^{\mathrm{C}^{n+1}}$ be an analytic set-valued selection of Lemma 1.1 and let

$$
R(z)=\left\{(w, u) \in \mathbf{C}^{2 n+1}: w \in K(z), u \in L(z, w)\right\}
$$

for $z \in \bar{G}$. Observe that $R$ is the composition of the multifunctions

$$
z \rightarrow\{z\} \times K(z), \quad(z, w) \rightarrow L(z, w),
$$

and so is analytic in $G$ by [11, Proposition 5.1(i), (ii)]. 
Since $\operatorname{gr}(L)$ is contained in the variety $\left(z-z^{*}\right) u_{1}+\left(w_{1}-w_{1}^{*}\right) u_{2}+\cdots+$ $\left(w_{n}-w_{n}^{*}\right) u_{n+1}=1$, therefore $\operatorname{pr}(h(\operatorname{gr}(R)))$, where pr: $\mathbf{C}^{2 n+2} \rightarrow \mathbf{C}^{n+1}$ denotes the standard projection, is disjoint with a neighbourhood of $\left(z^{*}, w^{*}\right)$.

By the countable covering argument we obtain now that there exists a sequence of annuli $P_{i}$ containing $\operatorname{gr}(K)$ and analytic multifunctions $L_{i}$ defined in $P_{i}$ such that if $R_{i}$ is defined as in (3.3), then

$$
\operatorname{gr}(K) \subset \operatorname{pr}\left(h\left(\operatorname{gr}\left(R_{i}\right)\right)\right) \subset \mathbf{C}^{n+1} \backslash U_{i}
$$

where $\left\{U_{i}\right\}$ is a covering of $\mathbf{C}^{n+1} \backslash \operatorname{gr}(K)$.

Define further for $(z, w) \in P_{1} \cap \cdots \cap P_{s}$ and for $z \in \bar{G}$ $\tilde{L}^{s}(z, w)=L_{1}(z, w) \times \cdots \times L_{s}(z, w), \quad \tilde{R}^{s}(z)=\left\{(w, u): w \in K(z), u \in \tilde{L}^{s}(z, w)\right\}$. Multifunctions $\tilde{L}^{s}$ are analytic by [11, Proposition 5.1(iii)], and then $\tilde{R}^{s}$ are analytic, in the same way that $R$ was.

By construction of $\tilde{L}^{s}$ and (3.4)

$$
\operatorname{pr}\left(h\left(\operatorname{gr}\left(\tilde{R}^{s}\right)\right)\right) \subset \operatorname{pr}\left(h\left(\operatorname{gr}\left(\tilde{R}^{s-1}\right)\right)\right) \cap \operatorname{pr}\left(h\left(\operatorname{gr}\left(R^{s}\right)\right)\right) \subset \mathbf{C}^{n+1} \backslash\left(U_{1} \cup \cdots \cup U_{s}\right)
$$

where pr denotes a suitable projection. Define

$$
X^{s}=\left\{(z, u): z \in \partial G, u \in \tilde{R}^{s}(z)\right\} .
$$

By Proposition $2.2 \operatorname{gr}\left(\tilde{R}^{s}\right) \subset h\left(X^{s}\right)$. Let $K^{s}$ be a multifunction with domain $G$ whose graph is the projection of the polynomial hull of $X^{s}$ onto $\mathbf{C}^{n+1}$. By (3.5) we get $\operatorname{gr}(K) \subset \operatorname{gr}\left(K^{s+1}\right) \subset \operatorname{gr}\left(K^{s}\right)$; the intersection of $\operatorname{gr}\left(K^{s}\right)$ equals $\operatorname{gr}(K)$. Q.E.D.

4. Consequences of the approximation theorem. The following theorem generalizes [11, Theorem III]. It will be applied in $\S 5$ to the study of analytic perturbations of the Taylor spectrum.

THEOREM 4.1. Let $G$ be a bounded planar domain and $K: G \rightarrow 2^{\mathrm{C}^{n}}$ an analytic multifunction with bounded graph. Then there exists a separable uniform algebra $A$ and functions $f, g_{1}, \ldots, g_{n} \in A$ such that

(i) $f\left(M_{A}\right) \backslash f\left(\partial_{A}\right)=G$, where $\partial_{A}$ denotes the Shilov boundary of $A$;

(ii) $g\left(f^{-1}(z)\right)=K(z)$ for every $z \in G$, where $g=\left(g_{1}, \ldots, g_{n}\right)$.

PROOF (SKETCH). We apply Theorem 3.1 as well as its proof. By construction the sets $\operatorname{gr}(K) \leftarrow h\left(X^{1}\right) \leftarrow h\left(X^{2}\right) \leftarrow \cdots \leftarrow h\left(X^{s}\right) \leftarrow \cdots$ considered with the natural projections, form an inverse system of compact sets whose limit can be identified with a compact subset $Y$ of $\mathbf{C}^{T}$, where $T$ is some countable index set. Then $Y$ is polynomially convex in $\mathbf{C}^{T}$ as the intersection of a family of (inverse images of) polynomially convex sets. (We consider polynomials in any finite number of variables.) Define $A$ to be the uniform closure of restrictions of all polynomials to $Y$, and let $f, g_{1}, \ldots, g_{n}$ be the coordinate functions $z, w_{1}, \ldots, w_{n}$. Then $\left(f, g_{1}, \ldots, g_{n}\right)(Y)=\operatorname{gr}(K)$ (by properties of $\left.X^{s}\right)$, which proves (ii). Now it is clear that the Shilov boundary of $A$ is contained in the inverse limit of the sets $X^{s}$ and so $f^{-1}(\partial G) \subset \partial_{A}$. Q.E.D.

We apply now Theorem 3.1 to disprove two plausible but false conjectures concerning polynomial hulls:

(1) If $Y=h(X)$ where $X \subset \partial D \times \mathbf{C}^{n}$ and $Y \neq X$, then $Y$ can be approximated by analytic varieties. 
(2) If $X, Y$ are as in (1) and if

$$
\tilde{Y}=\{(z, w): z \in \bar{D}, w \in \operatorname{co}(Y(z))\}
$$

where

$$
Y(z)=\left\{w \in \mathbf{C}^{n}:(z, w) \in Y\right\}
$$

("co"=convex hull), then $\tilde{Y}$ is polynomially convex.

EXAMPLE 4.2. Conjecture (1) is false. Consider any bounded analytic multifunction $K: D \rightarrow 2^{\mathrm{C}}$ with convex fibers $K(z)$ and examine the consequences of (1) for the multifunction $K$. Approximate $K$ by multifunctions $K^{s}(z)$ such that $\operatorname{gr}\left(K^{s}\right)=\operatorname{pr}\left(h\left(X^{s}\right)\right)$, with $X^{s}$ and $K^{s}$ satisfying conditions of Theorem 3.1. Let $Y^{s}=h\left(X^{s}\right)$ and define $Y^{s}(z)$ and $\tilde{Y}^{s}$ by (4.2) and (4.1) respectively. Then

$$
\operatorname{pr}\left(\tilde{Y}^{s}(z)\right)=\operatorname{co}\left(K^{s}(z)\right) \text {. }
$$

If (1) is true, for every neighbourhood $U$ of $h\left(X^{s}\right)$ in $\bar{D} \times \mathbf{C}^{m}$ there exists a closed analytic variety $M$ in $D \times \mathbf{C}^{m}$ contained in $U$. Consider $U$ containing $\operatorname{gr}\left(\tilde{Y}^{s}\right)$ and bounded in the direction $\{0\} \times \mathbf{C}^{m}$. By well-known facts $M$ is the graph of a finitely valued bounded analytic function $z \rightarrow\left\{a_{1}(z), \ldots, a_{n}(z)\right\}: D \backslash E \rightarrow \mathbf{C}^{m}$, where $E$ is a discrete sequence of isolated branching points. Then $n^{-1}\left(a_{1}(z)+\cdots+a_{n}(z)\right)$ defines an analytic function $a_{U}: D \rightarrow \mathbf{C}^{m}$ whose graph approximates $\tilde{Y}^{s}$ fairly well because of the convexity of $\tilde{Y}^{s}(z)$. Taking a suitable sequence of neighbourhoods $U$ and applying Montel's theorem to $\left\{a_{U}\right\}_{U}$ we find an analytic function $a^{s}: D \rightarrow \mathbf{C}^{m}$ such that $a^{s}(z) \in \tilde{Y}^{s}(z)$ for $z \in D$, and so by (4.3)

$$
f_{s}(z)=\operatorname{pr}\left(a^{s}(z)\right) \in \operatorname{co}\left(K^{s}(z)\right), \quad z \in D .
$$

Let $f(z)$ be the limit of some subsequences of $\left\{f_{s}\right\}$. By (3.1) and convexity of $K(z)$, $f(z) \in K(z)$ for all $z \in D$. Thus we have found a single-valued analytic selection from each bounded, convex-valued multifunction $K: D \rightarrow 2^{\mathbf{C}}$. This is, however, impossible by [6, Lemma 3.5], and so (1) fails.

REMARK 4.3. Conjecture (1) is a variant of the general problem posed by Alexander and Wermer concerning the possibility of approximating general singularity sets (which correspond to graphs of analytic multifunctions in our terminology) by analytic varieties $[\mathbf{1}$, Part I, Question 1]. Various positive results related to the latter problem were obtained in $[\mathbf{1}$, Parts I and II, 2, 15, 18], but the answer is negative in general, as shown in [6, Lemma 3.5].

In Example $4.2 n$ is rather large (but can be estimated). The case $n=1$ of conjecture (1) is still open.

EXAMPLE 4.4. Conjecture (2) is false. Let $\varphi$ be a subharmonic function in $D$. Then $K(z)=D(0, \exp \varphi(z)), z \in D$, is an analytic multifunction. Let $\varphi$ be such that $\{\varphi \equiv-\infty\}$ has limit points inside $D$. Then the only single-valued analytic selection from $K(z)$ over $D$ is $f(z) \equiv 0$.

We now apply to $K$ the same approximation procedure we did in Example 4.2. Thus the sets $X^{s}, Y^{s}, \tilde{Y}^{s}$ have the same properties and (4.3) holds. Conjecture (2) and Theorem 4.1 imply that $\tilde{Y}^{s} \cap\left(D \times \mathbf{C}^{m}\right)$ can be covered by graphs of analytic functions, and the same holds for the graph of $z \rightarrow \operatorname{co}\left(K^{s}(z)\right): D \rightarrow 2^{\mathrm{C}}$. Since $K(z)$, being convex, is the intersection of $\operatorname{co}\left(K^{s}(z)\right)$ (cf. (3.1)), Montel's theorem 
implies that $\operatorname{gr}(K)$ can be covered by graphs of analytic functions, which contradicts the initial observation.

In the last example $n$ could be rather large. The next example, found independently by $\mathrm{H}$. Alexander and J. Wermer (oral communication of Professor Wermer) and by the author, shows that conjecture (2) fails for $n=1$ as well.

EXAMPLE 4.5. Let $X=\left\{(z, w) \in \mathbf{C}^{2}:|z|=1, w^{2}=z\right\}$. Then $\tilde{Y}(z)=$ $\operatorname{co}\{\sqrt{z},-\sqrt{z}\}, z \in \bar{D}$, where $Y=\operatorname{gr}(K)$ and $\tilde{Y}$ is defined by (4.1). To show that $\tilde{Y}$ is not polynomially convex consider the set $Z=\left\{(z, w): z \in \bar{D},\left|w \pm \sqrt{w^{2}-z}\right| \leq 1\right\}$. One checks easily that sections $Z(z)$ are ellipses with foci $\sqrt{z},-\sqrt{z}$ for $|z|<1$ and line segments with endpoints $\sqrt{z},-\sqrt{z}$ for $|z|=1$. Furthermore $Z$ is the union of flat analytic discs $\{(z,(a+a z) / 2): z \in \bar{D}\}$, where $|a| \leq 1$, and so by the maximum property $Z \subset h(Z \cap(\partial D \times \mathbf{C}))=h(\tilde{Y} \cap(\partial D \times \mathbf{C}))$. Since $Z(0)=\left\{w:|w| \leq \frac{1}{2}\right\} \neq$ $\tilde{Y}(0)$, we conclude that $\tilde{Y}$ is not polynomially convex. (It can be shown that $Z$ is the polynomial hull of $\tilde{Y}$.)

We will now apply Theorem 4.1 to give a rather elementary proof of the following special case of Oka's theorem on the equivalence of pseudoconvex domains and domains of holomorphy.

COROLlaRY 4.6. Every bounded pseudoconvex domain $G$ in $\mathbf{C}^{2}$ is a domain of holomorphy.

Proof. Choose two discs $D, D_{1}$ in $C$ such that $D \times D_{1}$ contains $G$. Let $K: D \rightarrow$ $2^{\mathrm{C}^{n}}$ be the upper semicontinuous multifunction such that $\operatorname{gr}(K)=D \times \bar{D}_{1} \backslash G$. By [11, Theorem II] $K$ is analytic, and so by Theorem 4.1 (or Theorem III in [11]) there exist a uniform algebra $A$ and functions $f, g \in A$ such that $K(z)=g\left(f^{-1}(z)\right)$ for $z \in D$ and $f\left(M_{A}\right) \backslash f\left(\partial_{A}\right)=D$. In [16, Proof of Theorem 2, p. 15, and Lemma 2, p. 14] it was proven in an elementary way that the set

$$
U=\left\{(z, w) \in \mathbf{C}^{2}: z \in f\left(M_{A}\right) \backslash f\left(\partial_{A}\right), w \notin g\left(f^{-1}(z)\right)\right\}
$$

is a domain of holomorphy (for any uniform algebra $A$ and $f, g \in A$ ). Since $G$ is a union of connected open components of $U$, it is a domain of holomorphy itself. Q.E.D.

5. Analytic perturbations of the Taylor spectrum. It was shown in [11, Corollary 3.3 and Theorem 4] that analytic multifunctions in dimension one and multifunctions of the form $z \rightarrow \operatorname{sp}(T(z))$, where $T(z)$ is an analytic family of bounded operators, are locally the same. One way generalization to higher dimension was obtained by the author in $[\mathbf{1 4}]$. (Below $\operatorname{sp}\left(T_{1}, \ldots, T_{n}\right)$ denotes the joint spectrum defined in Taylor $[\mathbf{1 7}]$.)

THEOREM $5.1[\mathbf{1 4}]$. Let $T_{1}, \ldots, T_{n}: G \rightarrow B(X)$ be analytic operator-valued functions where $G$ is an open subset of $\mathbf{C}^{k}$ and $X$ is a complex Banach space. Assume that for each $\lambda \in G$ operators $T_{1}(\lambda), \ldots, T_{n}(\lambda)$ commute. Set $K(\lambda)=$ $\operatorname{sp}\left(T_{1}(\lambda), \ldots, T_{n}(\lambda)\right)$ for $\lambda \in G$. Then $K: G \rightarrow 2^{\mathrm{C}^{n}}$ is an analytic multifunction.

In this section we present a partial converse to this theorem when $k=1$ and for arbitrary $n$. The case of arbitrary $k$ seems to be open at present.

THEOREM 5.2. Let $K: G \rightarrow 2^{\mathrm{C}^{n}}$ be an analytic multifunction with bounded graph defined in a planar domain $G$. Then there exist analytic operator-valued 
functions $T_{j}: G \rightarrow B(H), j=1, \ldots, n$, where $H$ is a separable Hilbert space such that for each $\lambda \in G$ operators $T_{1}(\lambda), \ldots, T_{n}(\lambda)$ commute and

$$
K(\lambda)=\operatorname{sp}\left(T_{1}(\lambda), \ldots, T_{n}(\lambda)\right), \quad \lambda \in G .
$$

PROOF. The proof follows closely that of Theorem IV in $[\mathbf{1 1}, \S 6]$. We will recall the general argument and indicate the modifications that should be made.

Choose a separable uniform algebra $A$ and its elements $f, g_{1}, \ldots, g_{n}$ satisfying conditions of Theorem 4.1 relative to the given multifunction $K$. For this algebra $A$ we construct a separable Hilbert space $H$ and a representation $T: A \rightarrow B(H)$ in the same way as in [11, Lemma 6.1]. Namely, we choose a sequence of probability measures $m_{n}$ on $X$ (=Shilov boundary of $A$ ) representing a dense sequence of characters $\mu_{n}$ in $M_{A}$. We let $H_{n}$ be the completion of $A \mid X$ in $L^{2}\left(m_{n}\right)$ and $H$ the Hilbertian direct sum of all $H_{n}$ 's. For any $h \in A$ define $T h \in B(H)$ as the operator of multiplication by $h$. It was proven in [11, Lemma 6.1] that $T$ is an isometric isomorphism of algebra $A$ into $B(H)$ and that

$$
\sigma_{\pi}(T a) \subset a(X) \text { for } a \in A,
$$

where $\sigma_{\pi}$ denotes the approximate point spectrum.

Assertion. If $0 \in f\left(M_{A}\right) \backslash f(X)$, then

$$
\operatorname{sp}\left(\left(T g_{1}\right)^{\sim}, \ldots,\left(T g_{n}\right)^{\sim}\right)=\left\{z \in \mathbf{C}^{n}:(0, z) \in(f, g)\left(M_{A}\right)\right\},
$$

where $\left(T g_{j}\right)^{\sim}$ denotes the operator induced by $T g_{j}$ on the quotient space $H / \operatorname{Im}(T f)$, and $g=\left(g_{1}, \ldots, g_{n}\right)$.

(Note that by (5.1) the quotient space is a Banach space.) We prove first that if $(0, z) \in(f, g)\left(M_{A}\right)$ then

$$
(T f)(H)+\left(T g_{1}-z_{1} I\right)(H)+\cdots+\left(T g_{n}-z_{n} I\right)(H) \neq H .
$$

We argue as in [11, Lemma 6.1, p. 379, last paragraph]. Take the sequence $\left(x^{k}\right) \subset H$ defined there; it was proven that $\lim _{k}(T f)^{*} x^{k}=0$. The same argument shows that $\lim _{k}\left(T g_{j}-z_{j} I\right)^{*} x^{k}=0$. Since $\left\|x^{k}\right\|=1$, this proves (5.3), which in turn, by definition of Taylor spectrum [17], implies that $(0, z) \notin \operatorname{sp}\left(\left(T g_{1}\right)^{\sim}, \ldots,\left(T g_{n}\right)^{\sim}\right)$. To prove the reverse inclusion in (5.3), consider $(0, z) \notin(f, g)\left(M_{A}\right)$. Then the equation $\left[g_{1}-z_{1} I\right] h_{1}+\cdots+\left[g_{n}-z_{n} I\right] h_{n}=e$ is solvable in the quotient algebra $A /(f A)$. Since $H / \operatorname{Im}(T f)$ is a module over this quotient algebra, Lemma 1.1 in $[\mathbf{1 7}]$ implies that $z \notin \operatorname{sp}\left(\left(T g_{1}\right)^{\sim}, \ldots,\left(T g_{n}\right)^{\sim}\right)$. The Assertion is proved.

Set now $V(\lambda)=T f-\lambda I, S_{j}(\lambda)=T g_{j}, j=1, \ldots, n$, as at the beginning of the proof of Theorem IV in $[\mathbf{1 1}$, p. 381].

There we have defined a covering $\left\{G_{j}\right\}$ of $G$, a Hilbert space $H^{\prime}$, and analytic operator-valued functions

$$
R_{j}: G_{j} \rightarrow B\left(H, H^{\prime}\right), \quad U_{j}: G_{j} \rightarrow B\left(H^{\prime}\right) .
$$

These objects depended only on $V(\cdot)$ and we take them without change. They have the following properties:

$$
\begin{gathered}
U_{j}(\lambda)^{-1} \circ R_{j}(\lambda)=U_{k}(\lambda)^{-1} \circ R_{k}(\lambda), \quad \lambda \in G_{j} \cap G_{k}, \\
\operatorname{ker} R_{j}(\lambda)=\operatorname{Im} V(\lambda), \quad \lambda \in G_{j} .
\end{gathered}
$$

We conclude that functions $\left\{U_{j}^{-1} R_{j}\right\}$ define an analytic function $B: G \rightarrow B\left(H^{\prime}\right)$ such that $\operatorname{ker} B(\lambda)=\operatorname{Im} V(\lambda)$ and $\operatorname{Im} B(\lambda)=H^{\prime}$ for every $\lambda \in G$. Let $S_{j}(\lambda)^{\sim} \in$ 
$B(H / \operatorname{Im} V(\lambda))$ and $B(\lambda)^{\sim} \in B\left(H / \operatorname{Im} V(\lambda), H^{\prime}\right)$ be the operators induced by $S_{j}(\lambda)$ and $B(\lambda)$ respectively. Set

$$
T_{j}(\lambda)=B(\lambda)^{\sim} \circ S_{j}(\lambda)^{\sim} \circ\left(B(\lambda)^{\sim}\right)^{-1}, \quad \lambda \in G .
$$

By the Assertion $\operatorname{sp}\left(S_{1}(\lambda)^{\sim}, \ldots, S_{n}(\lambda)^{\sim}\right)=K(\lambda)$, and since the Taylor spectrum is preserved by simultaneous similarity (which follows directly from its definition $[\mathbf{1 7}])$, then $\operatorname{sp}\left(T_{1}(\lambda), \ldots, T_{n}(\lambda)\right)=K(\lambda)$ for $\lambda \in G$.

Of course $T_{1}(\lambda), \ldots, T_{n}(\lambda)$ commute and are bounded for each $\lambda \in G$. Finally, by $\left[11\right.$, Corollary 6.4] functions $T_{1}(\cdot), \ldots, T_{n}(\cdot)$ are analytic. Q.E.D.

REMARK. The construction of families $U_{j}(\lambda)$ given in [11] depended on the assumption that spaces $H / \operatorname{Im}(T f-\lambda I)$ are infinite-dimensional for $\lambda \in G$ and had to be modified otherwise. The author thanks Professor T. W. Gamelin for pointing out that these quotient spaces must be automatically infinite dimensional because of the way they are constructed. (Note: each $H_{n}$ is invariant for $T f-\lambda I$ and $(T f-\lambda I) H_{n} \neq H_{n}$ if $\lambda \in G$.)

\section{REFERENCES}

1. H. Alexander and J. Wermer, On the approximation of singularity sets by analytic varieties. Part I, Pacific J. Math. 104 (1983); Part II, Michigan Math. J. 32 (1985), 227-235.

2. __ Polynomial hulls with convex fiber, Math. Ann. 271 (1985), 99-109.

3. B. Aupetit, Analytic multivalued functions in Banach algebras and uniform algebras, Adv. in Math. 44 (1982), 18-60.

4. Geometry of pseudoconvex open sets and distributions of values of analytic multivalued functions, Proc. Conf. Banach Algebras and Several Complex Variables (F. Greenleaf and D. Gulick, eds.), Contemp. Math., vol. 32, Amer. Math. Soc., Providence, R. I., 1984.

5. B. Aupetit and J. Wermer, Capacity and uniform algebras, J. Funct. Anal. 28 (1978), 386-400.

6. B. Berndtsson and T. J. Ransford, Analytic multifunctions, the $\bar{\partial}$-equation and a proof of the corona theorem, Pacific J. Math (to appear).

7. R. Narasimhan, Introduction to the theory of analytic spaces, Lecture Notes in Math., vol. 25, Springer-Verlag, Berlin, Heidelberg and New York, 1966.

8. T. J. Ransford, Analytic multivalued functions, Doctoral thesis, Univ. of Cambridge, 1984.

9. __ Open mapping, inversion and implicit function theorems for analytic multivalued functions, Proc. London Math. Soc. (3) 49 (1984), 537-562.

10. - Interpolation and extrapolation of analytic multivalued functions, Proc. London Math. Soc. 50 (1985), 480-504.

11. Z. Slodkowski, Analytic set-valued functions and spectra, Math. Ann. 256 (1981), 363-386.

12. __ Analytic multifunctions, q-plurisubharmonic functions and uniform algebras, Proc. Conf. Banach Algebras and Several Complex Variables (F. Greenleaf and D. Gulick, eds.), Contemp. Math., vol. 32, Amer. Math. Soc., Providence, R. I., 1984.

13. __ Local maximum property and q-plurisubharmonic functions in uniform algebras, J. Math. Anal. Appl. (to appear).

14. _ Analytic perturbations of Taylor spectrum (to appear).

15. ___ Polynomial hulls with convex sections and interpolating spaces, Proc. Amer. Math. Soc. 96 (1986), 255-260.

16. __ Uniform algebras and analytic multifunctions, Accad. Naz. Lincei Rend. Cl. Sci. Ser. VIII 75 (1983), 9-18.

17. J. L. Taylor, A joint spectrum for several commuting operators, J. Funct. Anal. 6 (1970), 172-191.

18. J. Wermer, Green's functions and polynomial hulls, Proc. Conf. Banach Algebras and Several Complex Variables (F. Greenleaf and D. Gulick, eds.), Contemp. Math., vol. 32, Amer. Math. Soc., Providence, R. I., 1984.

Department of Mathematics, University of Illinois at Chicago, Box 4348, ChicAgo, Illinois 60680 